\title{
Reação de hipersensibilidade a colírios midriáticos: Um caso clínico
}

\section{Hypersensitivity to ophthalmic mydriatic agents: A case report}

Data de receção / Received in: 16/2/2020

Data de aceitação / Accepted for publication in: 13/4/2020

Rev Port Imunoalergologia 2021;29 (I):55-59

Ana Palhinha', Ana Margarida Romeira', Paula Leiria Pinto',2

I Serviço de Imunoalergologia, Hospital de Dona Estefânia, Centro Hospitalar Universitário de Lisboa Central (CHULC), EPE, Lisboa

${ }^{2}$ CEDOC, Integrated Pathophysiological Mechanisms Research Group, Nova Medical School, Lisboa

\section{RESUMO}

O uso crescente de medicação ocular levou ao aparecimento de um maior número de reações de hipersensibilidade $(\mathrm{RH})$ associadas à mesma. Estas são, na sua maioria, RH tipo IV e, mais raramente, imediatas. Os agentes envolvidos são diversos, desde substâncias ativas dos colírios midriáticos (CM), a substâncias inativas (conservantes). Apresenta-se o caso clínico de um doente com suspeita de RH imediata e não imediata a medicação ocular tópica (tropicamida $10 \mathrm{mg} / \mathrm{ml}$, fenilefrina $100 \mathrm{mg} / \mathrm{ml}$ e ciclopentolato $10 \mathrm{mg} / \mathrm{ml}$ ), cuja investigação incluiu realização de testes cutâneos por picada (TCP) e testes epicutâneos (TE) com os fármacos referidos. Os TCP foram positivos para ciclopentolato no imediato e para fenilefrina às 48 e 96 horas. Os TE foram positivos às 48 e 96 horas para fenilefrina. De acordo com estes resultados, assumiu-se $\mathrm{RH}$ a fenilefrina e ciclopentolato, sendo realizada prova de provocação ocular (PPO) com tropicamida, que o doente tolerou.

Palavras-chave: Colírios midriáticos, dermatite de contacto alérgica, medicação ocular, reações de hipersensibilidade.

\section{ABSTRACT}

The increasing use of ophthalmic medication has led to the emergence of a greater number of hypersensitivity reactions (HR) associated with it. These are mostly type IV HR and, rarely, immediate. The agents involved are diverse, from active substances such as mydriatic eye drops (CM) to inactive substances (preservatives). We report the case of a patient with suspected immediate and non-immediate HR to ophthalmic medication (tropicamide $10 \mathrm{mg} / \mathrm{ml}$, phenylephrine $100 \mathrm{mg} / \mathrm{ml}$ and cyclopentolate 
Ana Palhinha, Ana Margarida Romeira, Paula Leiria Pinto

$10 \mathrm{mg} / \mathrm{ml})$, whose investigation included skin prick tests (SPT) and patch tests (PT) with the mentioned drugs. SPT were positive for cyclopentolate, with immediate reaction, and for phenylephrine at 48 and 96 hours. The PT were positive at 48 and 96 hours for phenylephrine. According to these results, we assumed allergy to phenylephrine and cyclopentolate and an ocular provocation test was performed with tropicamide which was tolerated by the patient.

Keywords: Mydriatic eye drops, contact dermatitis, ocular medication, hypersensitivity reactions.

\section{INTRODUÇÃO}

A enorme variedade de medicação tópica ocular e o uso crescente da mesma conduziu não só a um aumento das reações adversas associadas a estes fármacos pela sua absorção sistémica, mas também a um número crescente de reações alérgicas ${ }^{1,2,3,4}$. Os agentes envolvidos são diversos, desde substâncias ativas, como os colírios mi-

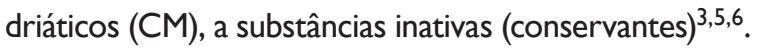

As reações de hipersensibilidade $(\mathrm{RH})$ a fármacos tópicos oftálmicos são, na sua maioria, reações tipo IV e, mais raramente, tipo $\mathrm{I}^{7,8}$. A dermatite de contacto alérgica (DCA), causa comum de dermatite periocular, é um exemplo de RH tipo IV, manifestando-se por eritema, pápulas ou vesículas e edema da região periorbitária, com prurido associado ${ }^{5}$. A fina epiderme periorbitária torna as pálpebras especialmente sensíveis à penetração da medicação tópica ocular, que constitui um importante grupo de haptenos ${ }^{5}$, podendo ser responsável por $20 \%$ dos casos de dermatite alérgica periocular ${ }^{4}$.

Os colírios midriáticos, como a fenilefrina, a tropicamida e o ciclopentolato, são fármacos frequentemente utilizados, separada ou concomitantemente, quer durante a realização de exames oftálmicos, quer em procedimentos cirúrgicos. A fenilefrina é responsável pela maior parte das reações alérgicas a colírios midriáticos ${ }^{1,5}$, datando o primeiro caso descrito de $1979^{1,8}$, sendo muito mais escassos os casos associados à tropicamida e/ou ciclopentolato ${ }^{4,7}$.

Apresentamos o caso clínico de um doente com suspeita de reações de hipersensibilidade a colírios midriáticos e a respetiva marcha diagnóstica efetuada.

\section{CASO CLÍNICO}

Doente de 82 anos, sexo masculino, com antecedentes pessoais de hipertensão, diabetes mellitus tipo 2, hipertrofia benigna da próstata e cataratas, sem história pessoal de atopia.

Em abril de 2017, imediatamente após aplicação de CM em consulta de Oftalmologia, iniciou quadro de eritema e edema conjuntival e palpebral, bilateral, muito pruriginoso, que terá durado cerca de 3 meses. Foi observado múltiplas vezes nesse período, tendo realizado vários ciclos de antibioterapia (ATB) e corticoide tópico. Em setembro de 2017, foi submetido a cirurgia a cataratas do olho esquerdo, sem reação. Em fevereiro de 2018, após consulta em que foram administrados CM, desenvolve quadro clínico semelhante ao anteriormente descrito, com necessidade de 3 ciclos de ATB e corticoide tópico, com resolução em cerca de 2 meses. Em maio de 2018 , cerca de 24 horas após cirurgia a cataratas do olho direito (OD), aparecimento de eritema e edema da pálpebra do OD, com prurido associado. Na sequência deste episódio foi observado em consulta de Imunoalergologia, sob terapêutica tópica com dexametasona, clorofenamina e flurbiprofeno, ainda com edema da pálpebra superior direita e hiperemia ocular objetiváveis.

Foram identificados os fármacos utilizados nas consultas e cirurgias descritas e todos eles correspondiam a CM - tropicamida $10 \mathrm{mg} / \mathrm{ml}$, fenilefrina $100 \mathrm{mg} / \mathrm{ml}$ e ciclopentolato $10 \mathrm{mg} / \mathrm{ml}$. Não foi possível fazer a correspondência entre os fármacos e as diferentes reações, sendo utilizado um a dois fármacos por episódio. 
Quadro I. Testes cutâneos por picada e epicutâneos com colírios midriáticos (diâmetro da pápula mm)

\begin{tabular}{|l|c|c|}
\hline \multicolumn{1}{|c|}{ Concentração } & Prick 15 min/48h/96h & $\begin{array}{c}\text { Patch 48h/96h } \\
\text { (+ positivo; - negativo) }\end{array}$ \\
\hline Controlo negativo (solução salina) & $0 / 0 / 0$ & - \\
\hline Controlo positivo (histamina) & $12 / 0 / 0$ & - \\
\hline Fenilefrina $100 \mathrm{mg} / \mathrm{ml}$ & $0 / 9 / 9$ & $+/+$ \\
\hline Tropicamida $10 \mathrm{mg} / \mathrm{ml}$ & $0 / 0 / 0$ & $-/-$ \\
\hline Ciclopentolato $10 \mathrm{mg} / \mathrm{ml}$ & $7 / 0 / 0$ & $-/-$ \\
\hline
\end{tabular}

Uma vez que as reações descritas eram imediatas e não imediatas, a investigação alergológica foi realizada através de testes cutâneos por picada (TCP) e testes epicutâneos (TE) com os fármacos referidos (Quadro I). Optamos por utilizar os fármacos na sua formulação comercial usada na consulta de oftalmologia, tal como descrito por outros autores na literatura ${ }^{8}$. As concentrações máximas dos três $C M$ utilizados foram testadas em cinco controlos (TCP e TE), sendo todos os resultados negativos.

Da avaliação alergológica realizada destacam-se os seguintes resultados: TCP positivos para ciclopentolato no imediato e constatação de positividade para fenilefrina às 48 e 96 horas aquando da leitura dos TE; TE positivos às 48 e 96 horas para fenilefrina. Posteriormente, foi realizada prova de provocação ocular (PPO) com tropicamida, que foi negativa.

Perante a positividade dos testes cutâneos e o padrão recorrente das reações com a reexposição aos fármacos, entendeu-se que não seria ético a realização de PPO com ciclopentolato nem com fenilefrina. Não foram testadas as substâncias inativas, uma vez que as presentes na tropicami$\mathrm{da}$, tolerada pelo doente, eram constituintes dos outros $\mathrm{CM}$.

\section{DISCUSSÃo}

A fenilefrina consiste num potente vasoconstritor, com efeito simpaticomimético (agonista $\alpha$-adrenérgico), extensamente usado não só como midriático, mas também como descongestionante nasa $\left.\right|^{8,9}$. Os principais efeitos secundários da sua aplicação ocular são visão turva, sensação de queimadura, lacrimejo, irritação conjuntival e edema da córnea; as reações sistémicas, como crise hipertensiva e taquicardia, são muito menos frequentes ${ }^{8}$. Está especialmente associada a RH tipo IV, mas existem também casos descritos de RH tipo I I,8. Atendendo às semelhanças imuno-histológicas entre a mucosa nasal e conjuntival, não é possível explicar a maior frequência de reações tipo IV após o uso de gotas oftálmicas, uma vez que a fenilefrina é usada em maiores concentrações e por períodos mais prolongados a nível nasal ${ }^{8}$. A sensibilização por via ocular parece correlacionar-se com o número de utilizações prévias ${ }^{9,10}$.

A fenilefrina pertence à família da fenilamina, que se divide em agentes derivados da fenilpropanolamina (efedrina, pseudoefedrina e norepinefrina) e agentes derivados da feniletanolamida (fenilefrina e epinefrina) 1,5. A reatividade cruzada $(R C)$ entre estes diferentes agentes simpaticomiméticos não está bem definida, mas existem casos descritos de RC entre agentes de diferentes grupos $^{1,2,8}$. Está descrito um risco potencial de RC da fenilefrina com o homólogo etilefrina, utilizado sistemicamente em crises hipotensivas, pelo que é recomendado precaução, devendo realizar-se TE com este agente ${ }^{8}$ pelo menos, previamente à sua utilização.

O ciclopentolato e a tropicamida são fármacos anticolinérgicos (antimuscarínicos) que produzem não só 
midríase, mas também cicloplegia, através do relaxamento do corpo ciliar e da íris ${ }^{3,5}$. A sua absorção sistémica causa taquicardia, disritmias auriculares, febre e flushing, broncodilatação, prolongamento do tempo de esvaziamento gástrico e alterações do sistema nervoso central, desde sedação a crise psicótica aguda ${ }^{3,7}$. A maioria das reações associadas a cicloplégicos tópicos são erupções cutâneas ou oculares, mas existe na literatura um caso de anafilaxia ao ciclopentolato em gotas oftálmicas numa criança de 4 anos ${ }^{7}$.

$\mathrm{Na}$ investigação de hipersensibilidade a colírios midriáticos, de acordo com a literatura disponível, os testes epicutâneos (TE) são considerados fundamentais na investigação de $\mathrm{RH}$ tipo IV, sendo também realizados frequentemente testes cutâneos por picada $(\mathrm{TCP})^{8}$. As concentrações ótimas, bem como as formas farmacêuticas utilizadas na realização de TE e TCP, não estão padronizadas ${ }^{4,5,6}$. Alguns autores defendem a utilização de gotas oculares na sua formulação comercial, uma vez que a sua concentração é não irritativa ${ }^{8}$. No entanto, como não existem concentrações validadas para os testes cutâneos com CM, utilizamos controlos para testar (TCP e TE) as concentrações máximas dos três CM utilizados, sendo todos os resultados negativos, sustentando a relevância dos nossos resultados.

De acordo com a literatura, existe um elevado número de falsos negativos nos TE. Este facto poderá ser explicado por diferentes hipóteses, nomeadamente a fina espessura da epiderme da pálpebra, que poderá facilitar a penetração antigénica, a diferente concentração de hapteno presente no produto comercial usado nos TE, uma possível rápida evaporação das gotas oftálmicas durante a realização dos $\mathrm{TE}$, ou ainda o facto de alguns conservantes dos colírios oftálmicos contribuírem para um aumento da penetração cutânea a potenciais alergénios $^{5}$. Assim, a prova de provocação ocular, na presença de testes cutâneos negativos, é fundamental para comprovar a tolerância ao fármaco e encontrar alternativas seguras para o doente ${ }^{5,6}$.
Quando vários agentes tópicos são suspeitos numa reação, uma prova de provocação positiva a um dos agentes não exclui a possibilidade de reação aos restantes, mesmo com TCP e TE negativos, pelo que todos devem ser testados através de $\mathrm{PPO}^{2}$.

Ainda que de acordo com a literatura (Landeck et al.), os conservantes sejam menos alergénicos que as substâncias ativas dos fármacos oftálmicos, pode ser necessário testar os mesmos ${ }^{4,5,6}$. É fundamental ter em conta que os TE realizados com as substâncias inativas presentes nos fármacos oftálmicos são frequentemente irritativos, devendo-se provavelmente à maior concentração usada, quando comparada com a dos colírios ${ }^{6}$. No nosso caso, os conservantes não foram testados, pois a tolerância do colírio de tropicamida permitiu excluir a hipersensibilidade aos mesmos.

Apesar das reações a colírios midriáticos serem pouco frequentes, e na sua maioria serem reações não imediatas, tendem a ocorrer em doentes que necessitam repetidamente deste tipo de fármacos, como se verificou no doente aqui referido.

No caso apresentado, a investigação efetuada foi fundamental para identificar os colírios responsáveis pelas várias reações que o doente teve, bem como para confirmar a tolerância à tropicamida. É, ainda, importante definir as concentrações ótimas e o tipo de formulação para realização dos testes cutâneos, atendendo a que não existem concentrações validadas. Por último, será útil definir protocolos de provocação ocular seguros, de forma a encontrar a melhor solução para os doentes.

\section{Conflito de interesses}

Os autores declaram que não existem conflitos de interesses.

\section{Contacto:}

Ana Patrícia Galamba Palhinha

Serviço de Imunoalergologia

Hospital de Dona Estefânia, CHULC

Rua Jacinta Marto, II69-045 Lisboa Lisboa

E-mail: palhinha.ana@gmail.com 


\section{REFERÊNCIAS}

I. Fernández D, Martínez R, Luaces E, Fernández A, García J, Varo F. Allergic contact conjuntivitis and cross-reaction between phenylephrine and epinephrine due to phenylephrine eye drops. Ann Allergy Asthma Immunol 2016;117;550-72.

2. Haroun-Díaz E, Ruíz-García M, De Luxán de la Lastra S, Pastor-Vargas C, De las Heras M, Sastre Domínguez J, et al. Contact dermatitis to both tropicamide and phenylephrine eye drops. Dermatitis 2014;25:149-50.

3. Gunaydin B, Yalcin Cok O. Hazards of topical ophthalmic drug administration. Trends in Anaesthesia and Critical Care 20II;1:3I-4.

4. Novitskaya ES, Dean SJ, Craig JP, Alexandroff AB. Current dilemmas and controversies in allergic contact dermatitis to ophthalmic medications. Clin Dermatol 201I;29:295-9.

5. Grey KR, Warshaw EM. Allergic contact dermatitis to ophthalmic medications: relevant allergens and alternative testing methods. Dermatitis 2016;27:333-47.
6. Zheng Y, Chaudhari PR, Maibach HI. Allergic contact dermatitis from ophthalmics. Cutan Ocul Toxicol 2017;36:317-20.

7. Tayman C, Mete E, Catal F, Akca H. Anaphylactic reaction due to cyclopentolate in a 4-year-old child. J Investig Allergol Clin Immunol 2010;20:347-8.

8. Bobadilla-González P, Pérez-Rangel I, García-Menaya JM; Sánchez-Veja S, Cordobés-Durán C, Zambonino-Carreiras MA. Type IV reaction due to phenylephrine administered nasally with cross-reactivity with ethylephrine. J Investig Allergol Clin Immunol 2011;21:69-72.

9. Madsen JT, Andersen KE. Phenylephrine is a frequent cause of periorbital allergic contact dermatitis. Contact Dermatitis 2015;73:64-5.

10. Veramme J, Zaeytijd J, Lambert J, Lapeere HI. Contact dermatitis in patients undergoing serial intravitreal injections. Contact Dermatitis 2016;74:18-21. 